(C) 2019 IEEE. Personal use of this material is permitted. Permission from IEEE must be obtained for all other uses, in any current or future media, including reprinting/republishing this material for advertising or promotional purposes, creating new collective works, for resale or redistribution to servers or lists, or reuse of any copyrighted component of this work in other works. 


\section{Synthesis of Dual-Input Single-Output DC/DC Converters}

\author{
Hamzeh Aljarajreh \\ Electrical and Data Engineering \\ University of Technology Sydney \\ Sydney, Australia \\ Email: hamzeh.aljarajreh@ieee.org
}

\author{
Dylan Dah-Chuan Lu \\ Electrical and Data Engineering \\ University of Technology Sydney \\ Sydney, Australia \\ Email:dylandclu@ieee.org
}

\author{
C. K. Tse \\ Electronic and Information Engineering \\ The Hong Kong Polytechnic University \\ Kowloon, Hong Kong \\ Email:cktse@ieee.org
}

\begin{abstract}
This paper presents a topological study using power flow diagrams to derive all possible basic and non-isolated doubleinput single-output (DISO) converters. Unlike most reported DISO converters with one bidirectional port, this paper considers up to two bidirectional ports. The paper focuses on providing a general guideline of all power flow combinations and corresponding converter configurations. After eliminating the impractical configurations due to their indirect connection to some ports and their multiple conversion stages, three converter configurations have been identified and corresponding circuit realizations are demonstrated.
\end{abstract}

\section{INTRODUCTION}

The increasing use of renewable energy sources such as solar energy, wind energy and fuel cell have made popular demand on power electronic converters [1]-[3]. These converters serve as an electrical interface among various renewable energy sources, energy storage units and output loads to perform efficient power conversion, effective power conditioning and fast control. This paper explores practical power electronic converters for harvesting energy, which could be used for the development of current and future DC distribution systems. Multiple-input single-output (MISO) converters are suitable for applications where an integration of multiple input energy sources is required to supply one output. DISO is the simplest form of MISO where two input sources are connected to power one output as in PV systems and hybrid vehicles. In DISO converter, input ports can be connected to a current source, a voltage source or a mix of both in series or parallel. The output port can be connected to a current or voltage load [4]. Therefore, different DISO converters have been presented to cover the needs of different applications such as photovoltaic hybrid system [5], fuel-cell hybrid power system [6] and hybrid wind-solar system [7]. Consequently, different topologies of possible DISO converters should be available to cater for various types of input sources and load ports.

The overall efficiency of the converter can be enhanced by controlling the power flow distribution and the switches locations [8]. In a normal configuration, the input and output ports are unidirectional. In some cases, these ports are bidirectional. Therefore, the arrangement of converters and control methods will have significant effects on performance and efficiency of the DISO converter. An interface which handles the regenerative power of electric vehicles is a good example for bidirectional output port in [9].

In this paper, a topological study on all possible power flow combinations and corresponding converter configurations is presented. The paper is organized as follows: In Section II, a brief review of power flow graphs is presented. In Section III, based on the power flow graph explained in literature review, power flow graphs are derived and presented for two bidirectional ports. In section IV, waveforms results are presented. Finally, conclusion is explained in section V.

\section{POWER Flow OF THREe-PORT CONVERTERS (TPC)}

Power flow graphs technique was presented in [10] and [11] to design power factor correction PFC converters. The technique is extended to deriving DISO converters with unidirectional output power, as reported in [12]. Power flow graphs provide a systematic approach to analysing power processing stages and direction among the power ports. Based on [12], this paper further considers three different power flow configurations. Firstly, DISO converters with two unidirectional input ports and one unidirectional output port. Secondly, DISO converters with one unidirectional input port, one bidirectional input port and one unidirectional output port. Thirdly, DISO converters with one unidirectional input port and two bidirectional ports. The analysis criteria for each one of these configurations are power flow subgraphs, power flow graphs, all possible configurations of DISO converters and finally, most practical configuration out of these configurations.

1) DISO converters with unidirectional input and output ports: It contains only two types of power flow subgraphs, as shown in Fig. 1. Type I is shown in Fig. 1(a) where power is transferred separately from one port to another port. However in Type II, power is transferred simultaneously from two ports to one port, as shown in Fig. 1(b).

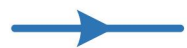

(a)

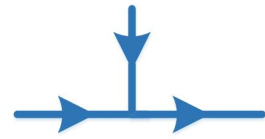

(b)
Fig. 1: DISO converters with unidirectional input and output ports power flow subgraphs. (a) Type I. (b) Type II. 
Based on the power flow subgraphs presented in Fig. 1, all possible power flow graphs were constructed in Fig. 2. All possible converters connections are explained in four different power flow graphs. Type I-I graph which includes two of Type I subgraphs. Then, a Type I-II graph includes one Type I subgraph and one Type II subgraph, that has two subtype inside, namely, Types I-IIA and I-IIB. Finally, Type II-II power flow graph includes two Type II subgraphs. One important point to mention is that Types I-IIA and I-IIB are exactly identical. Two converters will be arranged in proper paths of the power flow graphs to drive a complete DISO converters. Thirteen configurations of DISO is derived and shown in Fig. 3. Square boxes on the arrows represent a basic converter. All possible configurations for this input/output requirement are I-I, I-IIA, I-IIB, I-IIC, II-IIA, II-IIB, II-IIC, II-IID, IIIIE, II-IIF, II-IIG, II-IIH and II-III. Due to spatial symmetry, eight configurations only exist for DISO converters which are I-I, I-IIA, I-IIB, I-IIC, II-IIA, II-IIB, II-IIC, and II-IIF configurations.

2) DISO converters with one of the input ports being bidirectional: It has one of the input ports being bidirectional (for battery application for example) and one unidirectional output port. It contains three types of power flow subgraphs, as shown in Fig. 4. Type I and Type II are the same as in Fig. 1. Type III is shown in Fig. 4(c), power is transferred from one port to two ports simultaneously.

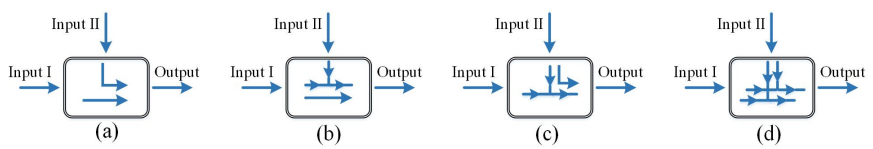

Fig. 2: Power flow graphs of Double-Input Single-Output converters with unidirectional input and output ports. (a) Type I-I. (b) Type I-IIA. (c) Type I-IIB. (d) Type II-II.

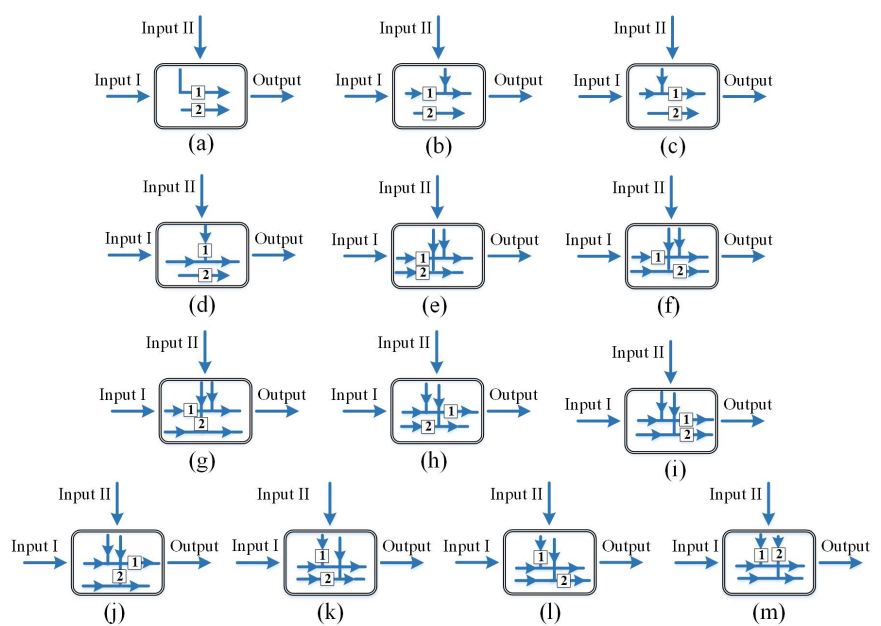

Fig. 3: All possible configurations of DISO with unidirectional input and output ports. (a) I-I. (b) I-IIA. (c) I-IIB. (d) I-IIC. (e) II-IIA. (f) II-IIB. (g) II-IIC. (h) II-IID. (i) II-IIE. (j) II-IIF. (k) II-IIG. (l) II-IIH. (m) II-III.
Based on the power flow subgraphs presented in Fig. 4, all possible power flow graphs were constructed in Fig. 5. All possible converter connections are explained in four different power flow graphs, namely, Type I-I, Type I-II, Type I-III and Type II-III. According to the theory in [10], the minimum number of converters to construct two subgraphs together is two simple converters. Therefore, Two converters will be arranged in proper paths of the power flow graphs to drive a complete DISO converters. Sixteen configurations of DISO are derived. All possible configurations for this input/output requirement are I-I, I-IIA, I-IIB, I-IIC, I-IIIA, I-IIIB, I-IIIC, IIA-IIIA, IIA-IIIB, IIA-IIIC, IIB-IIIA, IIB-IIIB, IIB-IIIC, IICIIIA, IIC-IIIB and IIC-IIIC. According to [11] out of these 16 configurations, only 5 of them have been used for practical implementation because it have more control of all ports which are Types I-I, I-IIA, I-IIB, I-IIIA \& I-IIIB. It has been rearranged in 2015 by [12] to be four configurations as in Fig. 6.

\section{DISO CONVERTER WITH TWO BIDIRECTIONAL PORTS}

In addition to the battery, a bidirectional port is useful in other applications such as port that handles the regenerative braking power from electric vehicles. Power flow subgraphs of DISO converter with a bidirectional output port are exactly the same as the unidirectional output port. It contains three types of power flow subgraphs, as shown in Fig. 4. All possible power flow graphs of Type I are presented in Fig. 7. All possible power flow graphs of 2 of Type I are presented in Fig. 8.

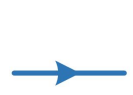

(a)

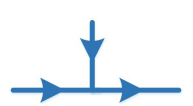

(b)

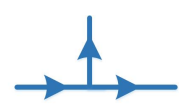

(c)
Fig. 4: DISO converters with one of the input ports being bidirectional (battery) power flow subgraphs. (a) Type I. (b) Type II. (c) Type III.

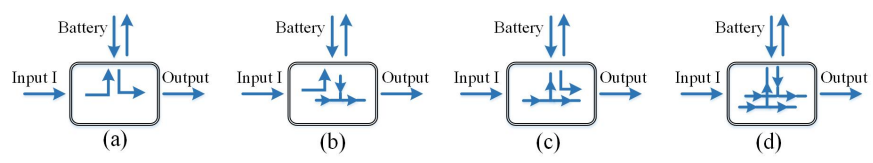

Fig. 5: Power flow graphs of Double-Input Single-Output converter with one of input ports is connected to battery as bidirectional port. (a) Type I-I. (b) Type I-II. (c) Type I-III. (d) Type II-III.
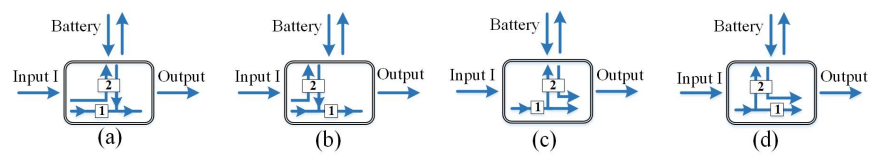

Fig. 6: Selected four configurations of DISO with one of input ports is connected to battery as bidirectional port. (a) I-IIA. (b) I-IIB. (c) I-IIIA. (d) I-IIIB. 
Then, all possible power flow graphs of 3 of Type I are shown in Fig. 9. However, these configurations require more converters, which is less efficient and costly. When power flow graphs of Type I and Type II are mixed, the resultant configurations are shown in Fig. 10. However, there are always two stages of converters. Finally only one power flow graph of 2 of Type II as shown in Fig. 11(a). It is worth noting that there are no possible configurations of Type II-III and Type III-III as bidirectional output port will not be considered in this power flow configuration. Two converters are arranged in proper paths of the power flow graphs to drive a complete DISO converters as in Fig. 11(b).

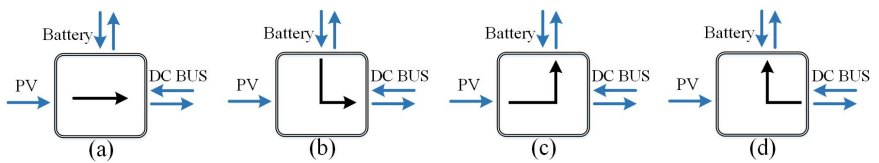

Fig. 7: All possible power flow graphs of Type I. (a) PV to DC bus. (b) Battery to DC bus. (c) PV to Battery. (d) DC bus to Battery.
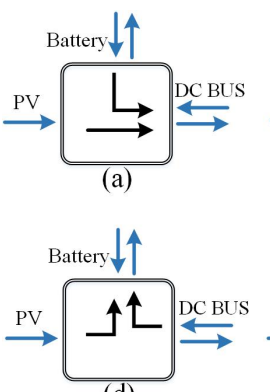

(d)

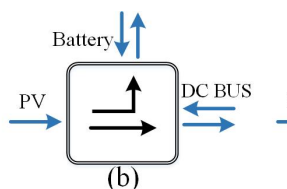

(b)

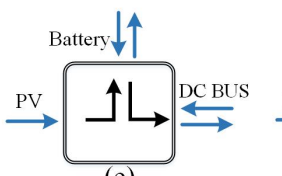

(e)
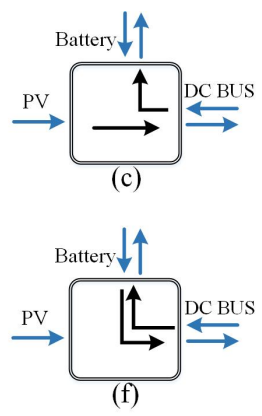

(f)
Fig. 8: All possible power flow graphs of two of Type I. (a) PV to DC bus \& Battery to DC bus. (b) PV to DC bus \& PV to Battery. (c) PV to DC bus \& DC bus to Battery. (d) PV to Battery \& DC bus to Battery. (e) PV to Battery \& Battery to DC bus. (f) Battery to DC bus \& DC bus to Battery.

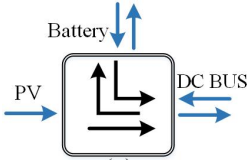

(a)

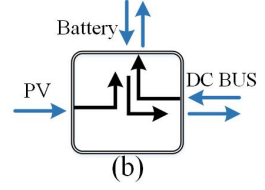

Fig. 9: Three of Type I configurations. (a) Battery to DC bus, DC bus to Battery \& PV to DC bus. (b) Battery to DC bus, DC bus to Battery \& PV to Battery.

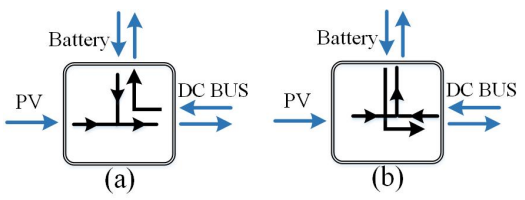

Fig. 10: Mixture of Type I and Type II configurations. (a) No direct connection between PV to Battery . (b) No direct connection between PV to DC bus.
Upon further inspection of each of those configurations, one of the ports are connected directly without converter which leaves it out of control. Therefore, only three configurations are useful as all ports are controlled as in Fig. 12. Power flow of Type II-IIA in Fig. 12(a) is used as an example. A possible converter configuration of Type II-IIA is presented in Fig. 13(a), two bi-directional buck/boost converters are used to realize box 1 and box 2 converters. A possible converter configuration of Type II-IIB is presented in Fig. 13(b). Finally, A possible converter configuration of Type II-IIC is presented in Fig. 13(c).

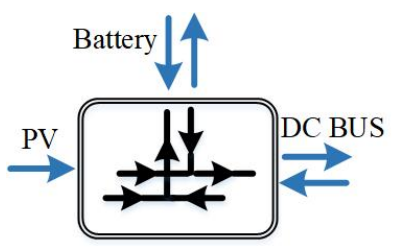

(a)
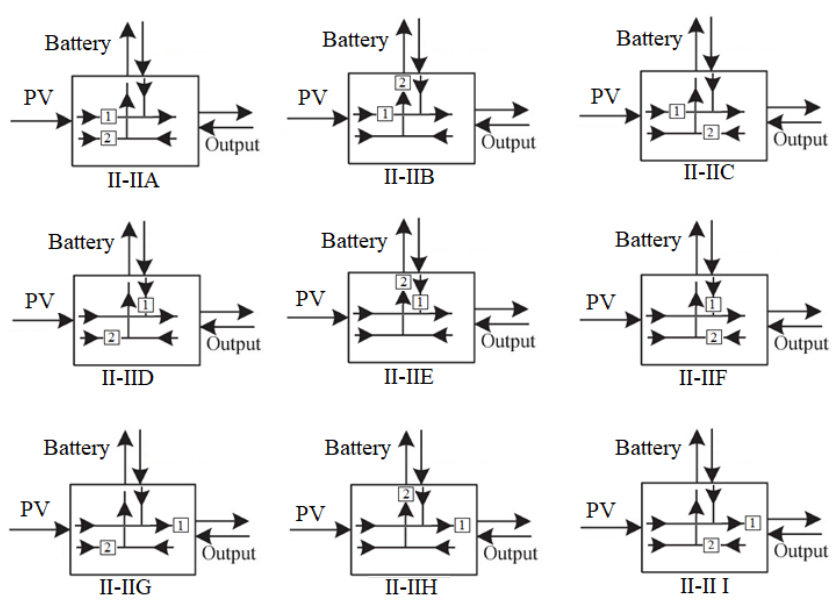

(b)

Fig. 11: All possible configurations of DISO. a) Power flow graph of 2 of Type II. b) All possible converter's paths of Type II-II.

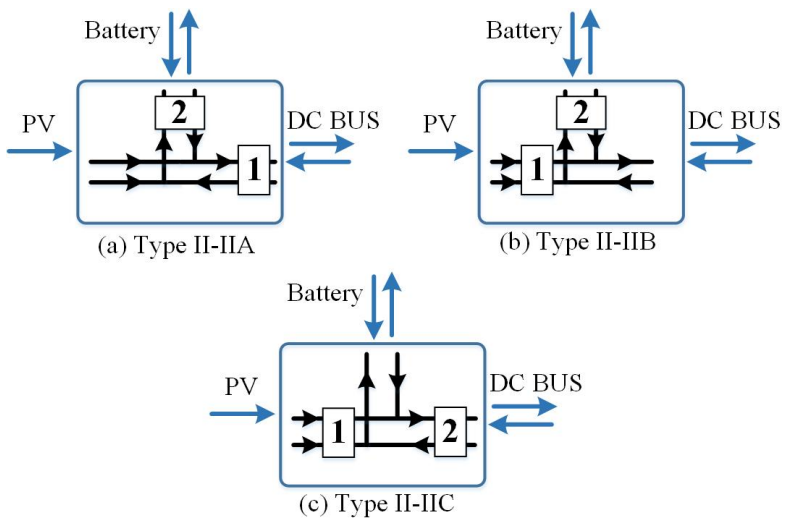

Fig. 12: Modified configurations of DISO with one bidirectional input port and bidirectional output port. (a) Type II-IIA. (b) Type II-IIB. (c) Type II-IIC. 


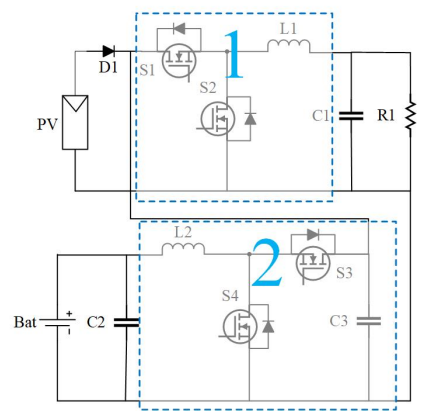

(a)

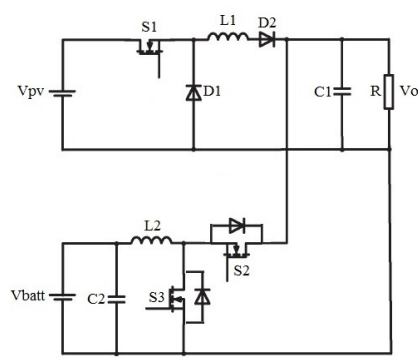

(b)

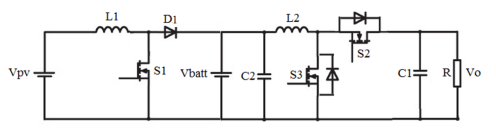

(c)

Fig. 13: Example converter circuits for a) Type II-IIA, b) Type II-IIB and c) Type II-IIC.

The efficiency of each architecture depends on how many power conversion stages are used. The advantage of the circuit in Fig. 13(a) is that PV has only one power conversion stage to DC bus or to battery. However, it uses two power conversions from battery to DC bus. The converter (II-IIB) in Fig. 13(b) has a buck converter between PV and DC bus and only one stage of power conversion between battery and DC bus. However, it needs two stages from PV to battery. Finally, The converter (II-IIC) in Fig. 13(c) has a two-stage converter which is less efficient from power conversion viewpoint. As shown in Fig. 13(a), a bidirectional buck-boost converter is used between PV and DC bus where also boost-buck converter can be used.

\section{EXPERIMENTAL RESUlTS}

A hardware prototype is built and tested based on Fig. 13(a). The switching patterns to achieve all seven modes of operation are shown in Table I. Even there are four switches in the converter, only two sets of PWM are required to implement all modes. Fig. 15 shows some key experimental waveforms. The order of these three traces from top view to bottom view are PWM1 gate driver signal, PWM2 gate driver signal and output voltage respectively.

TABLE I: Switching Look-up Table for Different Modes

\begin{tabular}{lllll}
\hline TPC with bidirectional output & S1 & S2 & S3 & S4 \\
\hline Mode 1 (PV to DC bus) & PWM1 & 0 & 0 & 0 \\
\hline Mode 2 (PV to battery and DC bus) & PWM1 & 0 & PWM2 & 0 \\
\hline Mode 3 (PV and battery to DC bus) & PWM1 & 0 & 0 & PWM2 \\
\hline Mode 4 (PV to battery) & 0 & 0 & PWM2 & 0 \\
\hline Mode 5 (battery to DC bus) & PWM1 & 0 & 0 & PWM2 \\
\hline Mode 6 (DC bus to battery) & 0 & PWM1 & PWM2 & 0 \\
\hline Mode 7 (PV and DC bus to battery) & 0 & PWM1 & PWM2 & 0 \\
\hline
\end{tabular}

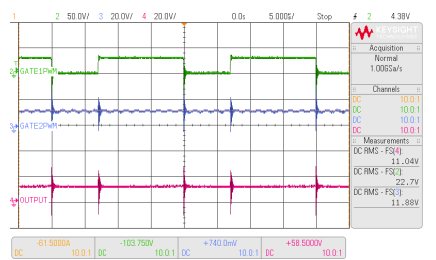

(a)

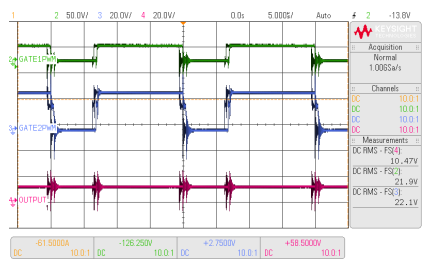

(c)

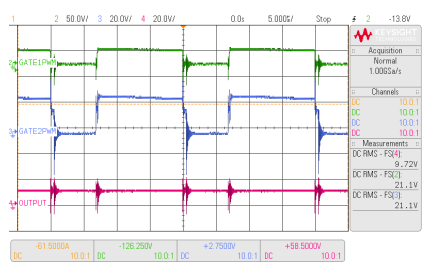

(e)

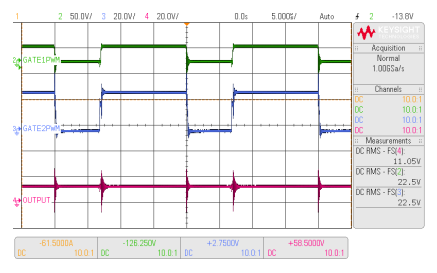

(b)

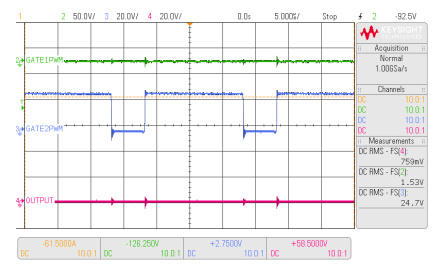

(d)

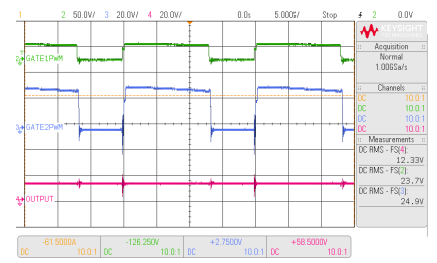

(f)

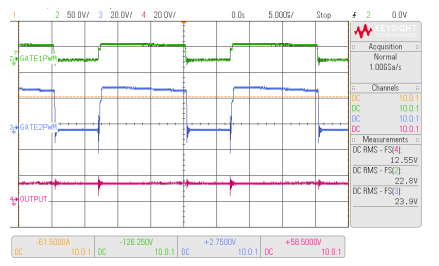

(g)

Fig. 14: Waveform results of Type II-IIA. a) Mode 1 (PV to DC BUS). b) Mode 2 (PV to DC BUS and Battery). c) Mode 3 (PV and Battery to DC BUS). d) Mode 4 (PV to battery). e) Mode 5 (Battery to DC BUS). f) Mode 6 (DC BUS to Battery). g) Mode 7 (DC BUS and PV to Battery).

\section{CONCLUSion}

This paper has presented a topological study to derive all possible double-input single-output (DISO) converters by using power flow graphs. Based on two conventional DISO converters, DISO with a bidirectional output port is explained in detail. Using Power Flow graphs minimised the number of converters and produced a more efficient design. The impractical configurations due to their indirect connection to some ports and their multiple conversion stages have been eliminated. Three converter configurations have been identified and corresponding circuit realizations are demonstrated.

\section{ACKNOWLEDGMENT}

This research was funded partially by the Australian Government through the Australian Research Council (Discovery Project No. DP180100129). 


\section{REFERENCES}

[1] S. Sinha and S. Chandel, "Review of recent trends in optimization techniques for solar photovoltaic-wind based hybrid energy systems," Renewable and Sustainable Energy Reviews, vol. 50, pp. 755-769, 2015.

[2] R. Rajesh and M. C. Mabel, "A comprehensive review of photovoltaic systems," Renewable and sustainable energy reviews, vol. 51, pp. 231248, 2015.

[3] N. Zhang, D. Sutanto, and K. M. Muttaqi, "A review of topologies of three-port dc-dc converters for the integration of renewable energy and energy storage system," Renewable and Sustainable Energy Reviews, vol. 56, pp. 388-401, 2016.

[4] Y. Huang and K. T. Chi, "Circuit theoretic classification of parallel connected dc-dc converters," IEEE Transactions on Circuits and Systems I: Regular Papers, vol. 54, no. 5, pp. 1099-1108, 2007.

[5] S.-K. Kim, J.-H. Jeon, C.-H. Cho, J.-B. Ahn, and S.-H. Kwon, "Dynamic modeling and control of a grid-connected hybrid generation system with versatile power transfer," IEEE transactions on industrial electronics, vol. 55, no. 4, pp. 1677-1688, 2008.

[6] K. Jin, X. Ruan, M. Yang, and M. Xu, "A hybrid fuel cell power system," IEEE Transactions on Industrial Electronics, vol. 56, no. 4, pp. 1212-1222, 2009.

[7] T. Hirose and H. Matsuo, "Standalone hybrid wind-solar power generation system applying dump power control without dump load," IEEE transactions on industrial electronics, vol. 59, no. 2, pp. 988-997, 2012.

[8] J. Sakly, A. B.-B. Abdelghani, I. Slama-Belkhodja, and H. Sammoud, "Reconfigurable dc/dc converter for efficiency and reliability optimization," IEEE Journal of Emerging and Selected Topics in Power Electronics, vol. 5, no. 3, pp. 1216-1224, 2017.

[9] T. Cheng, D. D.-C. Lu, and L. Qin, "Non-isolated single-inductor $\mathrm{dc} / \mathrm{dc}$ converter with fully reconfigurable structure for renewable energy applications," IEEE Transactions on Circuits and Systems II: Express Briefs, 2017.

[10] C. K. Tse, M. H. Chow, and M. K. Cheung, "A family of pfc voltage regulator configurations with reduced redundant power processing," IEEE Transactions on Power Electronics, vol. 16, no. 6, pp. 794-802, 2001.

[11] C. K. Tse, "Circuit theory of power factor correction in switching converters," International journal of circuit theory and applications, vol. 31, no. 2, pp. 157-198, 2003.

[12] P. Yang, C. K. Tse, J. Xu, and G. Zhou, "Synthesis and analysis of double-input single-output dc/dc converters," IEEE Transactions on Industrial Electronics, vol. 62, no. 10, pp. 6284-6295, 2015. 